Pacific Journal of Mathematic 


\title{
GROUPS WHOSE IRREDUCIBLE CHARACTER DEGREES ARE ORDERED BY DIVISIBILITY
}

\author{
R. Gow
}

\begin{abstract}
This paper concerns the class of finite groups whose complex irreducible character degrees can be linearly ordered by divisibility. It is known that such a group has a Sylow tower. By analyzing the structure of a group in the class whose order is divisible by just two primes, we are able to obtain information on the Sylow subgroups of any group in the class. We classify the two-prime groups in the class Except for certain exceptional pairs of primes, one of which is always 2. Such a group $G$ of order $p^{a} q^{b}$ either has a normal abelian Sylow $q$-subgroup or $H=G / O_{p}(G)$ has a non-abelian Sylow $q$-subgroup $Q$ and each $p$-element of $H$ induces a fixedpoint-free automorphism of $Q^{\prime}$.
\end{abstract}

Let $L$ denote the class of finite groups $G$ with the property that the degrees of the complex irreducible characters of $G$ can be linearly ordered by divisibility. The class $L$ was considered in two previous papers, [4] and [6], and the existence of a Sylow tower for any member of $L$ was shown in both papers. While we have not succeeded in describing $L$ purely in group-theoretic terms, the results of this paper indicate that the possible structure of a group in $L$ should be extremely limited.

We consider a non-abelian group $G$ in $L$ and let $\pi$ be the set of distinct primes dividing the degrees of the irreducible characters of $G$. Then if $\pi$ contains $r$ primes, it follows from [6], Theorem 2, that $G$ has a Sylow tower

$$
1 \triangleleft H_{r} \cdots \triangleleft H_{3} \triangleleft G
$$

where $H_{r}$ is a normal abelian Hall $\pi^{\prime}$-subgroup of $G$. We may consider the case where $r=1$ in the above description to be the trivial example of a group in the class $L$. The degrees of the irreducible characters of $G$ are all powers of some prime $p$ and $G$ has a normal abelian p-complement. Conversely, by a theorem of Ito, [3], Theorem 17. 10, p. 570, if $G$ has a normal abelian $p$-complement, the degrees of the irreducible characters of $G$ are all powers of $p$ and so $G \in L$.

It is our intention in this paper to analyze the structure of a group $G$ in $L$ whose order is divisible by precisely two primes. This analysis, although of a restricted nature, can be applied to an arbitrary group in $L$. For if $H_{i}$ is any of the normal subgroups of $G$ occurring in the Sylow tower described in the previous paragraph, $H_{i}$ is in $L$, 
and as $L$ is evidently factor-group-closed, our two-prime analysis may be applied to suitable factor-groups of the $H_{i}$. In particular our main theorem will yield information about the Sylow subgroups of any group in $L$.

1. Two-prime analysis. Let $G \in L$ and suppose that $|G|=p^{a} q^{b}$, where $p$ and $q$ are distinct primes. Suppose that $p$ divides $\chi(1)$ for each nonlinear irreducible character, $\chi$, of $G$. $G$ must have a normal Sylow $q$-subgroup $Q$. We will show that provided $p$ and $q$ are not certain exceptional primes, then there are only two possible structures for $G$. Let $O_{p}(G)$ be the largest normal $p$-subgroup of $G$. We note that $O_{p}(G)$ centralizes $Q$, and thus if $P$ is a Sylow $p$-subgroup of $G$, $Q$ admits $P / O_{p}(G)$ as a group of automorphisms. Using this notation we will prove

THEOREM 1. Let $G \in L$ and assume that $|G|=p^{a} q^{b}$ where $p$ and $q$ are distinct primes. Assume further that if $p=2, q$ is neither a Fermat nor a Mersenne prime, or if $p$ is a Mersenne prime, $q$ is not 2. Then we have either

(i) $Q$ is abelian or (ii) $Q$ is non-abelian, each element of $P / O_{p}(G)$ induces a fixed-point-free automorphism of $Q^{\prime}$, the derived group of $Q$, and $O_{p}(G)$ is abelian.

Thus we see that either the trivial situation occurs or $G / O_{p}(G)$ is almost a Frobenius group. The second theorem of this paper will show that, apart from the prime exceptions, we have completely classified two-prime groups in $L$. However, we have been unable to ascertain whether the prime exceptions described in Theorem 1 can give rise to exceptions to the theorem or whether the theorem holds for all pairs of primes. Before we begin the proof of Theorem 1, we state as a lemma a theorem due to Ito, [5], upon which our proof rests.

LEMMA 1. Let $G$ be a group in which $O_{p}(G)=1$ and suppose that $G$ has a normal nilpotent p-complement $H$. Then if one of the following holds

(i) G has odd order.

(ii) $H$ has even order and $p$ is not a Mersenne prime.

(iii) $p=2$ and $|H|$ is not divisible by any Fermat or Mersenne primes, $G$ has an irreducible character, $\chi$, of defect 0 modulo $p$ (that is, if $|G|=p^{a}|H|, p^{a}$ divides $\chi(1)$ ).

2. Proof of Theorem 1. We can assume that $Q$ is non-abelian. We will need the following facts about a solvable group $G$ : if $F(G)$ 
is the Fitting subgroup of $G$ and $\Phi(G)$ the Frattini subgroup of $G$, then $F(G / \Phi(G))=F(G) / \Phi(G)$ and $F(G) / \Phi(G)$ is the direct product of the minimal normal subgroups of $G / \Phi(G)$, [3], p. 277-9. In particular $F(G) / \Phi(G)$ is abelian. Our initial arguments will apply to $H=G / O_{p}(G)$. $H$ has a normal $p$-complement isomorphic to $Q$, so we may as well assume that $Q$ is a normal $p$-complement of $H$. Let us put $|H|=p^{c} q^{b}$.

Now $H / \Phi(H)$ has a normal abelian $p$-complement and so the degrees of its irreducible characters are powers of $p$. As $O_{p}(H)=1$, our remarks of the opening paragraph show that $O_{p}(H / \Phi(H))$ is also trivial. We can thus apply Lemma 1 to deduce that $H / \Phi(H)$ has an irreducible character, $\theta$, of degree $p^{c}$. Thus $H$ also has an irreducible character of degree $p^{c}$ and as $H \in L$, if $\chi$ is an irreducible character of $H$ with $q \mid \chi(1)$, then $p^{c} \mid \chi(1)$.

As we are assuming $Q$ is non-abelian, $Q$ has nonlinear irreducible characters. Let $\chi$ be a nonlinear irreducible character of $Q$ and let $T$ be the stabilizer of $\chi$ in $H$ (see [3], p. 569, 17.6b). By a theorem of Gallagher, [3], p. 572, there is an extension, $\psi$ of $\chi$ to $T$ and $\psi^{H}$ is an irreducible character of $H$, [3], p. 571. We have $\psi^{H}(1)=$ $\chi(1)|H: T|$. As $q$ divides $\chi(1)$, it also divides $\psi^{H}(1)$ and so by our result of the previous paragraph $p^{c}$ divides $\psi^{H}(1)$. It follows that $|G: T|=p^{c}$ and so $T=Q$. Thus each nonlinear irreducible character of $Q$ has $p^{c}$ conjugates in $H$. It is this statement which implies that, if $P_{1}$ is a Sylow $p$-subgroup of $H, P_{1}$ acts without fixed points on $Q^{\prime}$.

For, suppose $x \in P_{1}$ and has order $p$. We consider the action of $x$ on the elements and characters of $Q^{\prime}$. Only linear characters of $Q$ are fixed by $x$, for we have just seen that the stabilizer of a nonlinear irreducible character of $Q$ is $Q$ itself. By Corollary 2 of [2]. $x$ fixes no elements of $Q^{\prime}$ other than the identity. Returning to the general case of the group $G$, we see that $P / O_{p}(G)$ acts fixed-point-free on $Q^{\prime}$.

The final step of the argument is to show that $O_{p}(G)$ is abelian. We accomplish this by giving further consideration to the character $\theta$, previously constructed for $H$. This character can be thought of as a character of both $H$ and $G$. We first consider it as a character of $H$ and examine its restriction to $Q$. We have by Clifford's theorem, $\theta_{Q}=u\left(\lambda_{1}+\cdots+\lambda_{t}\right)$ where the $\lambda_{i}$ are a complete set of $H$-conjugate irreducible characters of $Q$ and $u$ is some integer. As the degree of $\theta$ is a power of $p$ and $Q$ is a $q$-group, each character $\lambda_{i}$ must be linear. If $S$ is the stabilizer of $\lambda_{1}$ in $H$, Gallagher's theorem shows that there is an extension $\mu$ of $\lambda_{1}$ to $S$, and $\theta$ has the form $(\mu \sigma)^{H}$ where $\sigma$ is an irreducible character of $S / Q . \quad$ As $\operatorname{deg} \theta=|H: Q|=p^{c}, \operatorname{deg} \sigma$ must equal $|S: Q|$. Thus $\sigma$ is an irreducible character of $S / Q$ whose degree equals the order of $S / Q$. This is only possible if $S=Q$ and so $\lambda_{1}$ has $p^{c}$ conjugates in $H$. Since $O_{p}(G)$ centralizes $Q$ in $G$, it must 
be in the stabilizer subgroup of $\lambda_{1}$ in $G$, and it follows from our deduction above that the stabilizer of $\lambda_{1}$ in $G$ must be $O_{p}(G) \times Q$. Considering $\theta$ as a character of $G$ now, we know there is an extension $\nu$ of $\lambda_{1}$ to $O_{p}(G) \times Q$ and $\nu^{G}=\theta$.

Gallagher's theorem implies that if we take any irreducible character $\psi$ of $O_{p}(G)$ and consider it as a character of $O_{p}(G) \times Q$, the induced character $(\nu \psi)^{G}$ will be irreducible. Now if $\psi(1)>1,(\psi \nu)^{G}$ has degree some power of $p$ greater than $p^{c}$. But we have already constructed characters of degree $p^{c} \times a$ power of $q$ in $H$ and these provide irreducible characters of the same degree in $G$. As $G \in L$, $\operatorname{deg}(\nu \psi)^{G}$ must divide $p^{c} q^{d}$, for some $d>0$, and this is impossible if $\operatorname{deg} \psi>1$. Thus deg $\psi=1$, and it now follows that, as all irreducible characters of $O_{p}(G)$ are linear, $O_{p}(G)$ is abelian.

3. A converse of Theorem 1 . We can prove a converse of Theorem 1 which will show, in particular, that modulo the prime exceptions, we have completely classified the two-prime groups in $L$.

THEOREM 2. Let $G$ be a group which possesses a normal $p$ complement $H$ and let $P$ be a Sylow subgroup of $G$. Suppose $O_{p}(G)$ is abelian and $P / O_{p}(G)$ acts in a Frobenius manner on $H^{\prime}$ (assumed distinct from 1). Suppose, in addition, that $H \in L$. Then $G \in L$.

Proof. $O_{p}(G)$ centralizes $H$ and hence if $\chi$ is an irreducible character of $H, O_{p}(G)$ is contained in the stabilizer of $\chi$. Let $\chi$ be a nonlinear character of $H$ and let $T$ denote the stabilizer of $\chi$ in $G$. Put $P_{1}=P \cap T$. Since $O_{p}(G) \leqq T, O_{p}(G) \cong P_{1}$. Now as $P_{1}$ fixes a nonlinear character of $H, P_{1}$ fixes a nontrivial conjugacy class of $H$, by Corollary 2 of [1], and hence fixes a nonidentity element of $H$. If $P_{1} \neq O_{p}(G)$, since $P_{1} / O_{p}(G)$ acts in a Frobenius manner on $H^{\prime}, P_{1}$ fixes no nonidentity element of $H^{\prime}$. But by Theorem 1 of [2], if $P_{1}$ fixes no nonidentity element of $H^{\prime}, P_{1}$ fixes only linear characters of $H$. Thus we must have $P_{1}=O_{p}(G)$.

By Gallagher's theorem, if $\psi$ denotes an extension of $\chi$ to $T=$ $H \cdot O_{p}, \chi^{T}=\sum_{i=1}^{s}\left(\psi \omega_{i}\right)$, where the $\omega_{i}$ are the irreducible characters of $T / H \cong O_{p}(G)$. The $\omega_{i}$ are all one-dimensional as $O_{p}(G)$ is abelian. The characters $\left(\psi \omega_{i}\right)^{G}$ are all distinct and irreducible, [3], p. 571, Theorem 17.11, and have degree $\operatorname{deg} \chi\left|P: O_{p}\right|$.

If $\lambda$ is a linear character of $H$, and $S$ is the stabilizer of $\lambda$, we apply Gallagher's theorem again to show that if $\lambda^{S}=\sum_{i=1}^{t} \nu_{i}(1)\left(\nu_{i} \mu\right)$, where $\mu$ is an extension of $\lambda$ to $S$, and the $\nu_{i}$ are the irreducible characters of $S / H$, then each character $\left(\mu \nu_{i}\right)^{G}$ is irreducible and has degree $\nu_{i}(1)|G: S|$. We put $P_{2}=P \cap S$, and again we have $O_{p}(G) \subseteq$ $P_{2}$. Since $S / H \cong P_{2}$, the degree of any irreducible character $\nu_{i}$ of 
$S / H$ divides $\left|P_{2}: O_{p}(G)\right|$, by Ito's theorem, [3], p. 570. Hence deg $\left(\nu_{i} \mu\right)^{G}$ divides $\left|P: P_{2}\right|\left|P_{2}: O_{p}\right|=\left|P: O_{p}\right|$. If the distinct degrees of the nonlinear character of $H$ are $a_{1}, \cdots, a_{r}$ where $a_{1}$ divides $a_{2}, a_{2}$ divides $a_{3}$ etc., the degrees of the characters of $G$ are $a_{1}\left|P: O_{p}\right|, \cdots, a_{r}\left|P: O_{p}\right|$, and powers of $p$ which divide $\left|P: O_{p}\right|$. Hence $G \in L$.

4. Notes on the theorems. In Theorem 1, apart from the prime exceptions, we have shown that either $Q$ is abelian or $P / O_{p}$ acts as a fixed-point-free automorphism group on $Q^{\prime}$. In the latter case, it is well known that if $p$ is an odd prime, $P / O_{p}$ must be cyclic, and if $p=2, P / O_{p}$ is cyclic or generalized quaternion. Thus, in particular, if both $p$ and $q$ are odd, either $Q$ is abelian or $P$ is an extension of an abelian group by a cyclic group. When we have an arbitrary group $G$ of odd order in $L$, our two-prime analysis can always be applied to the two-prime composition factors described in the introduction. We can deduce that for about half the primes $p$ dividing $|G|$, the Sylow $p$-subgroups of $G$ are either abelian or metabelian.

In Theorem 2, $H^{\prime}$ admits $P / O_{P}$ as a fixed-point-free group of automorphisms, and it follows from a theorem of Thompson, [3], p. 505 , Theorem 8.14, that $H^{\prime}$ is nilpotent. We believe that the structure of a group in $L$ whose derived group is nilpotent should be a restricted nature and analysis of this situation may provide possible inductive hypotheses for investigating the structure of an arbitrary group in $L$.

\section{REFERENCES}

1. G. Glauberman, Correspondence of characters for relatively prime operator groups, Canad J. Math., (1968), 1465-88.

2. R. Gow and J. F. Humphreys, Normal p-complements and irreducible representations, submitted to J. London Math. Soc.

3. B. Huppert, Endliche Gruppen, Springer, Berlin, 1967.

4. I. M. Isaacs and D. S. Passman, A characterization of groups in terms of the degrees of their irreducible characters, Pacific J. Math., 24 (1968), 467-510.

5. N. Ito, Note on the characters of solvable groups, Nagoya Math. J., 39 (1970), 23-28.

6. J. G. Thompson, Normal p-complements and irreducible characters, J. Algebra, 14 (1970), 129-34.

Received July 5, 1974.

Carleton University, Ottawa 



\title{
PACIFIC JOURNAL OF MATHEMATICS
}

\author{
EDITORS
}

RICHARD ARENS (Managing Editor)

University of California

Los Angeles, California 90024

R. A. Beaumont

University of Washington

Seattle, Washington 98105
J. DugundJI

Department of Mathematics University of Southern California Los Angeles, California 90007

D. Gilbarg and J. Milgram

Stanford University

Stanford, California 94305

\section{ASSOCIATE EDITORS}

E. F. BECKENBACH

B. H. NeUManN

F. WOLF

K. YosHIDA

\section{SUPPORTING INSTITUTIONS}

UNIVERSITY OF BRITISH COLUMBIA

GALIFORNIA INSTITUTE OF TECHNOLOGY

UNIVERSITY OF CALIFORNIA

MONTANA STATE UNIVERSITY

UNIVERSITY OF NEVADA

NEW MEXICO STATE UNIVERSITY

OREGON STATE UNIVERSITY

UNIVERSITY OF OREGON

OSAKA UNIVERSITY
UNIVERSITY OF SOUTHERN CALIFORNIA

STANFORD UNIVERSITY

UNIVERSITY OF TOKYO

UNIVERSITY OF UTAH

WASHINGTON STATE UNIVERSITY

UNIVERSITY OF WASHINGTON

AMERICAN MATHEMATICAL SOCIETY NAVAL WEAPONS CENTER 


\section{Pacific Journal of Mathematics}

\section{Vol. 57, No. $1 \quad$ January, 1975}

Keith Roy Allen, Dendritic compactification ......................... 1

Daniel D. Anderson, The Krull intersection theorem ..................... 11

George Phillip Barker and David Hilding Carlson, Cones of diagonally dominant

matrices .............................................

David Wilmot Barnette, Generalized combinatorial cells and facet splitting ........

Stefan Bergman, Bounds for distortion in pseudoconformal mappings ............

Nguyên Phuong Các, On bounded solutions of a strongly nonlinear elliptic

equation ...............................................

Philip Throop Church and James Timourian, Maps with 0-dimensional critical

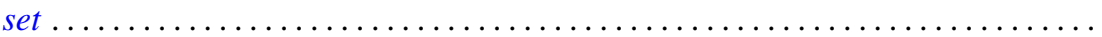

G. Coquet and J. C. Dupin, Sur les convexes ubiquitaires ................. 67

Kandiah Dayanithy, On perturbation of differential operators............... 85

Thomas P. Dence, A Lebesgue decomposition for vector valued additive set

functions .............................................. 91

John Riley Durbin, On locally compact wreath products.................. 99

Allan L. Edelson, The converse to a theorem of Conner and Floyd ............. 109

William Alan Feldman and James Franklin Porter, Compact convergence and the

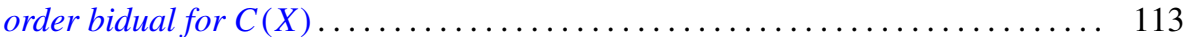

Ralph S. Freese, Ideal lattices of lattices......................... 125

R. Gow, Groups whose irreducible character degrees are ordered by divisibility ... 135

David G. Green, The lattice of congruences on an inverse semigroup ............ 141

John William Green, Completion and semicompletion of Moore spaces .......... 153

David James Hallenbeck, Convex hulls and extreme points of families of starlike and close-to-convex mappings .................................. 167

Israel (Yitzchak) Nathan Herstein, On a theorem of Brauer-Cartan-Hua type ...... 177

Virgil Dwight House, Jr., Countable products of generalized countably compact spaces ............................................ 183

John Sollion Hsia, Spinor norms of local integral rotations. I . . . . . . ......... 199

Hugo Junghenn, Almost periodic compactifications of transformation

semigroups....................................

Shin'ichi Kinoshita, On elementary ideals of projective planes in the 4-sphere and oriented $\Theta$-curves in the 3 -sphere ............................ 217

Ronald Fred Levy, Showering spaces............................ 223

Geoffrey Mason, Two theorems on groups of characteristic 2-type............. 233

Cyril Nasim, An inversion formula for Hankel transform ................... 255

W. P. Novinger, Real parts of uniform algebras on the circle ................ 259

T. Parthasarathy and T. E. S. Raghavan, Equilibria of continuous two-person

games............................................... 265

John Pfaltzgraff and Ted Joe Suffridge, Close-to-starlike holomorphic functions of several variables ....................................... 271

Esther Portnoy, Developable surfaces in hyperbolic space .................. 281

Maxwell Alexander Rosenlicht, Differential extension fields of exponential type . . . 289

Keith William Schrader and James Lewis Thornburg, Sufficient conditions for the

existence of convergent subsequences ........................... 301

Joseph M. Weinstein, Reconstructing colored graphs .................. 307 